\title{
Surfactant modulated interaction of hydrophobically modified ethoxylated urethane (HEUR) polymers with impenetrable surfaces
}

Mervat Shafik Ibrahim a,b, Sarah Rogers c , Najat Mahmoudy c, Martin Murray d, Agnieszka Szczygiel d, Beth Green ${ }^{d}$, Bruce D. Alexander a and Peter C. Griffiths ${ }^{a}$

a Faculty of Engineering and Science, University of Greenwich, Medway Campus, Chatham Maritime, Kent ME4 4TB, UK

b Pharmaceutics Department, Faculty of Pharmacy, Modern Science and Arts University,26 July Mehwar Road intersection with Wahat Road, Cairo, Egypt

c Science and Technology Facilities Council, ISIS Facility, Rutherford Appleton Laboratory, Didcot, Oxfordshire OX11 OQX, UK

d AkzoNobel, Wexham Road, Slough, Berkshire, SL2 5DS, UK

\section{Corresponding Author}

Peter Griffiths

Email: P.Griffiths@greenwich.ac.uk

Telephone: $+44(0) 2083319927$

Keywords: HEUR, Latex, SDS, solvent relaxation NMR, PGSE-NMR, rheology, SANS.

Submitted to J.C.I.S. 


\section{Abstract}

\section{Hypothesis}

The presence of surfactant modulates the surface-chemistry-specific interaction of hard colloidal particles (latex) with HEUR polymers, principally through introducing a preferential solution interaction rather than a competitive surface interaction; addition of surfactant leads to a preponderance of polymer/surfactant solution complexes rather than surface-bound complexes.

\section{Experiments}

A range of model formulations comprising a hexyl end-capped urethane polymer ( $\mathrm{C}_{6}-\mathrm{L}$ $\left.\left(E_{100}-\mathrm{L}\right) 9-\mathrm{C}_{6}\right)$, sodium dodecylsulfate (SDS) and a series of polystyrene-butylacrylate latices (PS-BA-L) have been characterised in terms of rheology, particle surface area (solvent relaxation NMR), polymer conformation (small-angle neutron scattering) and solution composition to build up a detailed picture of the distribution of the HEUR in the presence of both surfactant and latex.

\section{Findings}

There is very weak adsorption of $\mathrm{C}_{6}-\mathrm{L}-\left(\mathrm{EO}_{100}-\mathrm{L}\right) 9-\mathrm{C}_{6}$ to only the most hydrophobic latex surface studied, an adsorption that is further weakened by the addition of low levels of surfactant. Macroscopic changes in the hydrophobic latex system may be interpreted in terms of bridging flocculation at low polymer concentrations. No adsorption of $\mathrm{C}_{6}$ - $\mathrm{L}$-(EO100L) $9-\mathrm{C}_{6}$ is observed in the case of hydrophilic surfaces. In most cases, the observed behaviour of the ternary system (polymer/surfactant/particle) is highly reminiscent of the binary (polymer/surfactant) system at the appropriate composition, suggesting that the polymer/surfactant solution interaction is the dominant one. 


\section{Introduction}

Water-based latex dispersions are used as film formers in a range of formulations (e.g. paints, tablet coatings) (1-3). Hydrophobically modified ethoxylated urethane (HEURs) are ethylene oxide-urethane block copolymers, widely used to control the rheological profile of latex dispersions. However, polymers with such complex architectures interact with other formulation components, including surfactants.

The nature of the adsorption between HEUR and latex is sensitive to the hydrophobicity of the latex surface as both the HEUR hydrophobic end-groups and urethane linkers can adsorb on the hydrophobic surface of latex $(4,5)$. With decreasing hydrophobicity (e.g. incorporation of acrylic and methacrylic acid or 2-hydroxyethyl methacrylate (HEMA) monomers), only the polymer hydrophobic end-groups adsorb to the surface (6), and ultimately fewer end-groups adsorb at higher levels of hydrophilic monomer incorporation into the particles (7).

Ou-Yang et al. studied by dynamic light scattering (DLS) the conformation of HEUR adsorbed to polystyrene latex as a function of HEUR concentration (8). At low concentration, the authors argued that the end-groups and urethane linkers adsorb to the latex surface forming a pancake-like structure. However, at higher concentrations the urethane linkers desorb and only the hydrophobic end-groups remain adsorbed forming a brush-like structure. Generally, the viscosity builds in these particulate formulations due to the presence of a network of associating polymers, mediated through polymer bridging between particles forming a dynamic space-filling network. Additionally, the increase in viscosity also arises due to increases in the effective volume of the particles due to HEUR adsorption. The architecture of the polymer affects the viscosity and performance of the HEUR/latex dispersion, as increasing the polymer hydrophobic end-group length strengthens the adsorption leading to viscosity increases (9).

Two models for the possible arrangement of HEURs and particles in particulate formulations have been proposed. Pham et al. considered HEUR/latex interactions as a number of idealised configurations assuming that one hydrophobic end-group adsorbs on the latex surface, and the second end-group is adsorbed to: (a) the same latex particle ("loop"), (b) another latex particle ("bridge"), or (c) the end-group hydrophobic aggregate of another HEUR polymer ("micelle" or "network") (10). Beshah refined this model, based on a greater association of the hydrophobic moieties with the particle surface (6), Figure 1, though in the 
presence of surfactant or at higher HEUR concentrations, the distinction in polymer configuration in the two models is less (6).
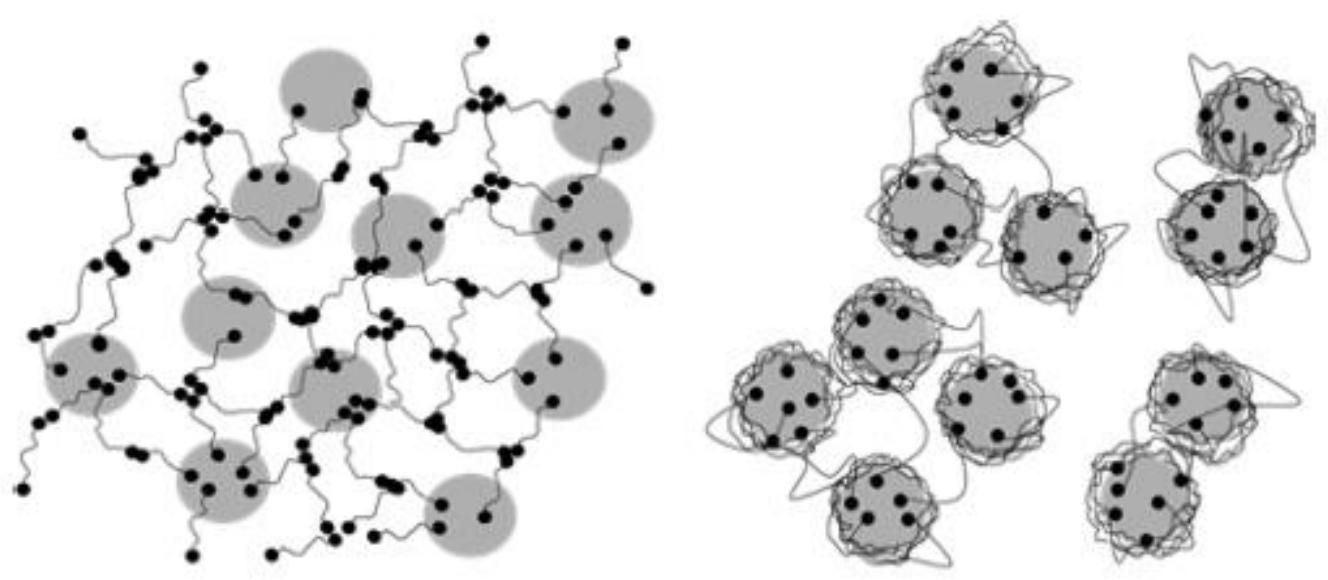

Figure 1. Schematic illustrating (a) original and (b) revised model conformation of the HEUR in presence of latex in paint formulation. Reproduced with permission from Beshah et al. (6). The particles are represented by the larger grey spheres, whereas the smaller black spheres depict the hydrophobic end-groups of the polymer.

In terms of the rheology, the shear thinning behaviour of the particulate dispersion is a consequence of either or both (i) the re-distribution of bridges and loops, thus the network structure is disrupted (6) and (ii) the desorption of the polymer molecules from the latex surface (11). A series of SANS experiments suggested that the adsorbed layer thins under shear (12), and that the adsorption in these system is weak, inducing particle aggregation at low polymer concentration as a consequence of the inclusion of SDS (13).

In a three-component model system comprising polymer/latex/surfactant, various interactions can occur. The relative affinity of surfactant for the polymer or latex is likely to determine whether or not the polymer is desorbed from the latex surface, and the mechanism of desorption. Polymer desorption from the latex surface in the presence of surfactant can be due to either direct competition for adsorption sites on the surface or through polymer/surfactant solution complexation.

Pisarcik et al. measured the viscosity of $1 \mathrm{wt} \%$ hydrophobically modified hydroxyethyl cellulose (HM-HEC) with a range of SDS concentrations at a fixed concentration of polystrene latex (PS-L) (14). The viscosity increases upon addition of latex at CSDS < CMC, the critical micelle concentration. The latex provides cross-linking points in the polymer network, hence the viscosity increase. At CSDS > CMC, the viscosity drops as the HM-HEC is desorbed from the PS-L surface as a result of the solubilisation of the polymer hydrophobic 
segments in SDS micelles, hence the cross-linking effect of the latex becomes negligible. The diffusion coefficient measured by DLS of PS-L in HM-HEC/PS-L mixtures increases as a function of SDS concentration.

Lauten et al. used DLS to study the change of the hydrodynamic radius $(R H)$ of $0.01 \%$ hydrophobically modified ethylhydroxyethyl cellulose and $0.001 \mathrm{wt} \%$ PS-L mixtures in the presence of a range of SDS concentrations (5-50 mmolal) $(15,16)$. The $R_{H}$ of HM-EHEC/PS$L$ mixture decreases, reflective of HM-EHEC desorption, explained by the binding of SDS to HM-EHEC increasing the hydrophilicity of the complex. Hence, the complex favours the bulk phase rather than adsorption to PS-L.

Mahli et al. studied the effect of 0.25 and $0.6 \mathrm{wt} \%$ SDS on the adsorption of HEUR on methyl methacrylate-methacrylic acid latex (MMA-MAA-L) (17). The concentration of the desorbed HEUR was determined by ultraviolet spectroscopy (UV) as a function of SDS. The SDS displaces all the adsorbed HEUR on the latex surface at $0.35 \mathrm{wt} \%$, whilst the nonionic surfactant nonylphenol ethoxylate-12 (NPE12) partially desorbed the HEUR from latex surface. Worthy of note, is that the affinity of SDS to HEUR is higher than non-ionic surfactants as the former interacts with hydrophobes and PEO backbone, whereas the latter interacts with the hydrophobic end-groups only (18-21). Therefore, the SDS is more capable of solubilising the polymer in the bulk, hence polymer desorption from the latex surface occurs.

Glass et al. studied the viscosity of HEUR at concentrations of 2.5 and $0.5 \mathrm{wt} \%$ with $25 \mathrm{wt} \%$ MAA-MMA-L (120 d.nm) (5).The addition of SDS at $0.3 \mathrm{wt} \%$ showed a drop in the viscosity of the polymer/latex mixture, which was correlated to the desorption of HEUR from the latex particle surface due to site competition with SDS.

In contrary to the conclusions drawn by Glass et al., Hulden suggested that the HEUR does not adsorb to latex in presence of SDS due to HEUR/surfactant interaction (9). The HEUR adsorbs to methyl methacrylate-butyl acrylate latex (MMA-BA-L) in presence of a non-ionic surfactant (nonylphenol ethoxylate-10) NPE-10 but not in presence of SDS. Since NPE10 can displace the SDS from the MMA-BA-L surface, the lack of HEUR adsorption can be explained by solution complexation of HEUR and SDS. Chatterjee et al. postulated that the SDS does not competitively desorb the HEUR from latex surface, in agreement with Hulden (12). However, the SDS micelles provide additional surface for the HEUR end-hydrophobe adsorption, hence the HEUR hydrophobes desorb from the latex surface. 
Ma et al. used UV spectroscopy to determine the amount of non-ionic surfactant NPE12 adsorbed to the latex after removal of the particles from the mixture by centrifugation and measuring the unadsorbed surfactant concentration in the supernatant (22). The competitive adsorption of the HEUR and surfactant has been studied by measuring the amount of surfactant liberated in solution as a function of HEUR concentration. A $\mathrm{C}_{12} \mathrm{HEUR}$ could not desorb the NPE12 from the latex surface; however, a $\mathrm{C}_{18}$ HEUR desorbed the surfactant.

Clearly, HEUR/latex interactions are dependent on many factors (e.g. latex surface chemistry, particle size, polymer architecture, concentration). In this paper, the ternary mixtures of the HEUR/SDS/latex have been studied with two latices of different surface chemistry, hence hydrophobicity, in an attempt to identify which of the various interactions is the key interaction and hence control the rheological properties of formulation.

\section{Material and methods}

\section{Materials}

Sodium persulphate ( $>99 \%$, Aldrich), d8-styrene ( $>98 \%$, Fischer Scientific), h-butylacrylate (>99\%, Aldrich), polystyrene-butylacrylate latex (diameter $=173 \mathrm{~nm}, \mathrm{PDI} 0.03)$ (AkzoNobel), acrylic acid stabilised polystyrene-butylacrylate latex (diameter $=154 \mathrm{~nm}$, PDI 0.03, acid level $3 \%$ ) (AkzoNobel), sodium dodecylsulphate (SDS) (Aldrich, no impurity observed), deuterated sodium dodecylsulphate ( $\mathrm{d}_{25}$-SDS) (ISIS deuteration facility), Hydroin buffer $\mathrm{pH}$ 9 (Aldrich), deionized water (18 $\mathrm{M} \Omega \mathrm{cm}$, Purite Select deionizer) and deuterium oxide (99.9\%, Aldrich) were used as received.

Hydrophobically modified ethoxylated urethane (HEUR) polymers are generally synthesised in two steps: (a) reaction between poly(ethylene glycol) (PEG) and the urethane linker, 4,4'diisocyanatodicyclohexylmethane $\left(\mathrm{H}_{12} \mathrm{MDI}\right)$ is used for the polymers studied here, through step polymerization technique that yields an ethoxylated urethane pre-polymer and subsequently (b) reacting this prepolymer with alcohol to provide the hydrophobic end caps, Error! Reference source not found.. The polymer used here is a commercial sample, obtained from Dow, herein denoted $\mathrm{C}_{6}-\mathrm{L}-\left(\mathrm{EO}_{100}-\mathrm{L}\right) 9-\mathrm{C}_{6}$ (Dow), where $\mathrm{L}$ is the urethane linker, $\mathrm{EO}_{100}$ the 100 monomer unit ethylene oxide block and a $\mathrm{C}_{6}$ hexyl alkyl end-group, and has been used as supplied. 


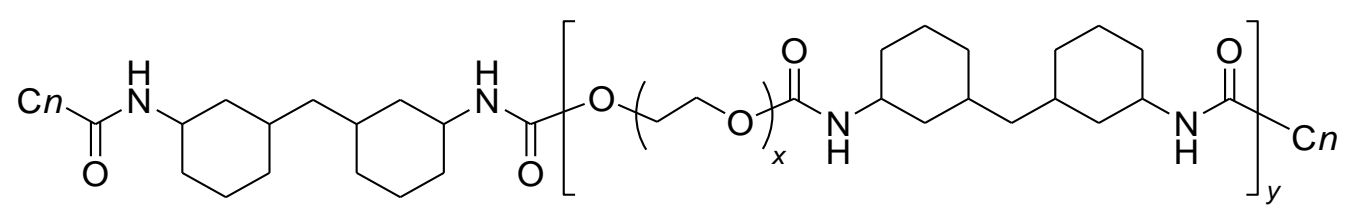

Table 1. Schematic illustration for the HEUR polymer studied in this paper where $n=6, x=100$, and $y=9$.

\section{Synthesis of deuterated latex particles}

The polymerization was carried out on a $50 \mathrm{~g}$ scale in a stirred, 5-port round bottom flask, under a nitrogen atmosphere, thermostatted at $70^{\circ} \mathrm{C}$. Half the initiator (sodium persulphate) was dispensed into the reaction vessel which contained $0.8 \mathrm{wt} \%$ aqueous SDS solution. The polymerisation reaction was initiated upon dropwise addition of the monomers ( $\mathrm{d}_{8}$-styrene, butylacrylate). The remaining second half of the initiator was added once half the monomer had been dispensed. The reaction was terminated by cooling the reaction vessel to room temperature. The sample was filtered using glass wool, and any unreacted monomer and SDS were removed by repeated centrifugation / re-suspension in $\mathrm{D}_{2} \mathrm{O} / \mathrm{H}_{2} \mathrm{O}$ mixtures. The particle concentration calculated from dry weight analysis was $20 \mathrm{wt} \%$. The diameter of the particles was measured by dynamic light scattering (zetasizer Nano-ZS, Malvern) as 160 nm with PDI 0.067.

\section{Methods}

Free SDS was removed from the (unreacted monomer free) experimental latices supplied by AkzoNobel by repeated centrifugation and re-suspension in the appropriate solvent, and dynamic light scattering was used to detect aggregation. All the HEUR/latex and HEUR/SDS/latex samples were prepared in the appropriate solvent according to the experiment and mixed using a hula-mixer for 24 hours before measurement.

\section{Nuclear Magnetic Resonance techniques}

\section{Solvent relaxation NMR}

HEUR/latex and HEUR/SDS/latex mixtures were dissolved in Hydroin buffered $\mathrm{H}_{2} \mathrm{O}$ at $\mathrm{pH}$ 9. Experiments were carried out at $25{ }^{\circ} \mathrm{C}$ on a bench-top Acorn XIGO Nanotools spectrometer. A Carr-Purcell-Meiboom-Gill (CPMG) sequence was used with a spacing of $0.5 \mathrm{~ms}$, between the $90^{\circ}$ and $180^{\circ}$ pulse, and a recycle delay of at least 5 times the spin-lattice relaxation time between consecutive scans to ensure full recovery of the 
magnetisation. Typically, 2118 data points were collected for each scan, and the signal was averaged over four scans for each sample. Data were fitted to a single exponential on the instrument software.

\section{Pulsed-Gradient Spin-Echo Nuclear Magnetic Resonance (PGSE-NMR)}

HEUR/latex and HEUR/SDS/latex mixtures were dissolved in Hydroin buffered $\mathrm{D}_{2} \mathrm{O}$ at $\mathrm{pH}$ 9. Experiments were carried out at $25^{\circ} \mathrm{C}$ on a $400 \mathrm{MHz}$ Bruker FT NMR spectrometer. A stimulated echo sequence was used, in which the diffusion time $(\Delta)$ was set to $800 \mathrm{~ms}$, the duration $(\delta)$ of the gradient pulses was held constant at $1 \mathrm{~ms}$ and their intensity $(G)$ varied from 5 - $800 \mathrm{G} \mathrm{cm}^{-1}$. Typically, 16 scans were accumulated over 32 gradient steps. Selfdiffusion coefficients were extracted by fitting the peak intensities $(I$ to Equation 1 for the peaks at $3.75 \mathrm{ppm}$ (EO) where $l_{0}$ is signal intensity in absence of gradient pulses, $D_{\mathrm{s}}$ the diffusion coefficient, $\gamma$ the gyromagnetic ratio of protons $(23,24)$.

$$
I=I_{0} e^{-D_{s} \gamma^{2} G^{2} \delta^{2}\left(\Delta-\frac{\delta}{3}\right)}
$$

Equation 1

\section{Neutron Scattering}

SANS measurements were carried out at $25^{\circ} \mathrm{C}$ on the SANS 2D instrument (ISIS spallation Neutron Source, Oxfordshire, UK). Neutron wavelengths spanning 2-14 $\AA$ were used to access a $Q$ range of 0.005 to $3 \AA^{-1}(Q=4 \pi \sin (\theta / 2) / \lambda)$ (25) with a fixed sample-detector distance of 4 and $2.4 \mathrm{~m}$ for the rear and front detector respectively. Temperature control was achieved through the use of a thermostatted circulating bath pumping fluids through the base of the sample changer, which allowed the experiment to be run at $25 \pm 0.5^{\circ} \mathrm{C}$. Samples were contained in UV-spectrophotometer grade $1 \mathrm{~mm}$ path length quartz cuvettes (Hellma). The scattering data were normalized for the sample transmission and the incident wavelength distribution, corrected for instrumental and sample backgrounds using a quartz cell filled with $\mathrm{D}_{2} \mathrm{O}$ (this also removes the incoherent instrumental background arising from vacuum windows), and corrected for the linearity and efficiency of the detector response using the instrument specific software package. The data were put onto an absolute scale using a well characterised, partially deuterated PS-L blend standard sample.

The scattering data were normalized for the sample transmission and the incident wavelength distribution, corrected for instrumental and sample backgrounds using a quartz cell filled with $\mathrm{D}_{2} \mathrm{O}$ (this also removes the incoherent instrumental background arising from vacuum windows), and corrected for the linearity and efficiency of the detector response 
using the instrument specific software package. The data were put onto an absolute scale using a well characterised, partially deuterated PS-L blend standard sample.

In the contrast match experiment, $d$-styrene and h-butylacrylate monomers were used to synthesize partially deuterated latex particles referred to as d-PS-h-BA-L. The d-PS-h-BA-L was diluted to $3 \mathrm{wt} \%$ in the scattering experiment with the appropriate solvent $\left(\mathrm{D}_{2} \mathrm{O}\right.$ or $\left.\mathrm{H}_{2} \mathrm{O}\right)$ to make different ratios of $\mathrm{H}_{2} \mathrm{O}: \mathrm{D}_{2} \mathrm{O}$ to find experimentally the match point of the latex, Figure S.1. After detecting the match point, conventionally found where the $\sqrt{I(Q)}=0$, and hence $\Delta \rho=0$, the HEUR and latex mixtures were prepared in the correct ratio of $\mathrm{D}_{2} \mathrm{O}: \mathrm{H}_{2} \mathrm{O}$ and the scattered intensity was recorded. In this contrast, only the $\mathrm{C}_{6}-\mathrm{L}-\left(\mathrm{EO}_{100}-\mathrm{L}\right)_{9}-\mathrm{C}_{6}$ contributes to the scattering intensity. The same contrast match experiment for HEUR/d-PS$\mathrm{h}-\mathrm{BA}-\mathrm{L}$ has been replicated in presence of SDS at two concentrations 0.1 and $1 \mathrm{wt} \%$. A mixture of $\mathrm{h} / \mathrm{d}$-SDS was used to match the scattering length density of the surfactant to the solvent, hence the scattering contribution arises only from the $\mathrm{C}_{6}-\mathrm{L}-\left(\mathrm{EO}_{100}-\mathrm{L}\right) 9-\mathrm{C}_{6}$.

\section{Rheology}

The rheology of mixtures of HEUR/PS-BA-L and HEUR/SDS/PS-BA-L have been examined in a matrix of experiments where the HEUR and SDS concentrations were held constant at $5 \mathrm{wt} \%$ and $0,0.1$ and $1 \mathrm{wt} \%$ respectively, and the latex concentration was varied $(0.5,3$, and $5 \mathrm{wt} \%$ ) in Hydroin buffered water, $\mathrm{pH}$ 9. The samples were measured using TA instrument rheometer AR-2000ex, with a $2^{\circ} / 40 \mathrm{~mm}$ cone and plate geometry at $25^{\circ} \mathrm{C}$. Shear profiles were recorded at $25^{\circ} \mathrm{C}$ for a shear range of $1-1000 \mathrm{~s}^{-1}$, with an integration time of 5 $\mathrm{s}$, and delay time of $5 \mathrm{~s}$.

\section{Results and discussion}

The interaction of the HEUR $\mathrm{C}_{6}-\mathrm{L}-\left(\mathrm{EO}_{100}-\mathrm{L}\right)_{9}-\mathrm{C}_{6}$ with two polystyrene-butylacrylate latices (PS-BA-L) has been studied in the absence and presence of SDS. Whilst the $\mathrm{C}_{6}-\mathrm{L}-\left(\mathrm{EO}_{100-}\right.$ L) ${ }_{9}-\mathrm{C}_{6} / \mathrm{PS}-\mathrm{BA}-\mathrm{L}$ system is of primary focus, selected experiments were conducted with the less hydrophobic latex AA-PS-BA-L for comparison, the latter with a $3 \%$ incorporation of acrylic acid. Changes in the polymer self-diffusion coefficient were studied in the presence and absence of latex to track changes in the unadsorbed polymer aggregates; solvent relaxation NMR was used to determine changes in the particle surface area; neutron scattering "contrast match" experiments to probe polymer conformations and finally, rheology to provide an indirect measure of polymer location. 


\section{Interaction of HEUR/PS-BA-L mixtures}

Solvent relaxation NMR is sensitive to surface area due to equilibrium of the water molecules adsorbed at the particle surface and the bulk phase. An increase in surface area leads to a decrease in the measured spin-spin relaxation time $T_{2}$, more conveniently expressed as its reciprocal the relaxation rate , $\left(R=\frac{1}{T_{2}}\right)$, normalized to the free solvent, $\left(R_{2 \mathrm{sp}}=\frac{R_{2}}{R_{2}^{\circ}}-1\right)$. Since $R_{2 \text { sp }}$ shows a linear dependence on surface area Figure S.2, any decrease in $R_{2 \text { sp }}$ of a suspension at fixed particle concentration reflects a loss of surface area (or equivalently, a reduction in the level of dispersion of the particles).

The dependence of $R_{2 \text { sp }}$ on the concentration of $\mathrm{C}_{6}-\mathrm{L}-\left(\mathrm{EO}_{100}-\mathrm{L}\right)_{9}-\mathrm{C}_{6}-$ in the absence of latex - is illustrated in Figure 2. $R_{2 s p}=0$ for most of the polymer range, only at the highest polymer concentrations does $R_{2 s p}>0$.The relatively minor increase in $R_{2 s p}$ at higher polymer concentrations is due to the increase in solution viscosity which restricts the motion of the solvent molecules (26).

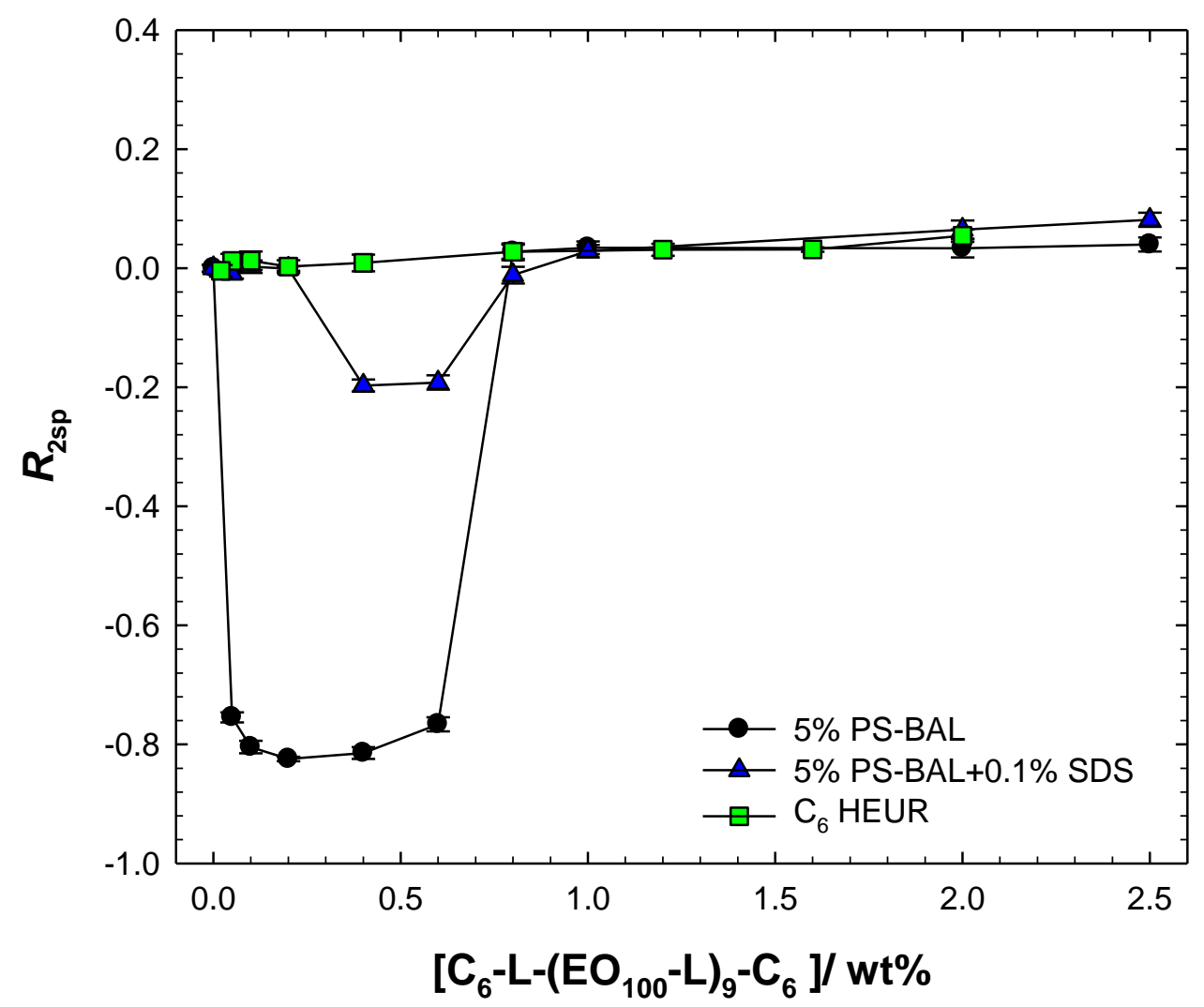

Figure 2. Specific relaxation rate $R_{2 s p}$ of the solvent in aqueous solutions containing $\mathrm{C}_{6}-\mathrm{L}-\left(\mathrm{EO}_{100}-\mathrm{L}\right)_{9}-\mathrm{C}_{6}$ as a function of its concentration in absence (squares) and presence of $5 \mathrm{wt} \%$ PS-BA-L (black circles), and $0.1 \mathrm{wt} \%$ SDS $/ 5$ wt $\%$ PS-BA-L (triangles) in Hydroin buffered water, $\mathrm{pH}$ 9. R2sp of HEUR/PS- 
BA-L mixtures are corrected relative to bare particle, and HEUR as a function of its concentration relative to water. Measurements were carried out at $25{ }^{\circ} \mathrm{C}$. The solid lines are guides for the eye. The error bars correspond to one standard deviation around the mean, derived from 5 repeat measurements on duplicate samples.

In the presence of a fixed concentration of the latex particles, for the majority of the concentration range $C_{\text {polymer }}>0.6 \mathrm{wt} \%, R_{2 s p}$ is equal to that of the polymer solution. For $0<$ $C_{\text {polymer }}<0.6 \mathrm{wt} \%, R_{2 \mathrm{sp}}$ is negative, reflective of the loss of surface area i.e. aggregation of particles,. Above $C_{\text {polymer }}>0.6$ wt\%, stability of the HEUR/latex dispersion returns, which is due to the ability of the polymer to associate in solution at those concentrations, presumably where one hydrophobic end-group is adsorbed to the latex surface and the other is in a polymer hydrophobic aggregate. The phase behaviour of $\mathrm{C}_{6}-\mathrm{L}-\left(\mathrm{EO}_{100}-\mathrm{L}\right)_{9}-\mathrm{C}_{6} / \mathrm{PS}-\mathrm{BA}-\mathrm{L}$ agrees with observations of Kostansek (27), Reuvers (28), and Jenkins (29) where the phase separation was correlated to bridging flocculation, and re-stabilisation induced by HEUR hydrophobe association in aqueous phase.

To date, few $T_{2}$ studies have been deployed on these systems. Uemura et al. (30) reported on the adsorption of a HEUR to PS-L. A strong adsorption of the HEUR to the latex was indicated by enhancement of the observed $\mathrm{T}_{2}$ where more than $50 \%$ of the polymer was bound. In the study by Uemura et al. there is a greater affinity of the polymer for the latex, leading to a significant enhancement in the $\mathrm{T}_{2}$ relaxation. By contrast, in our system the weak affinity of the polymer for the latex led to bridging flocculation at low polymer concentration.

The addition of $0.1 \mathrm{wt} \%$ SDS to the HEUR/latex mixtures in this study showed the same relaxation curve trend with a less intense dip and a narrower aggregation window relative to the binary mixture of $\mathrm{C}_{6}-\mathrm{L}-\left(\mathrm{EO}_{100}-\mathrm{L}\right) 9-\mathrm{C}_{6} / \mathrm{PS}-\mathrm{BA}-\mathrm{L}$, Figure 2.

The changes in the specific relaxation rate and equivalently, the surface area of the dispertsion of $5 \mathrm{wt} \%$ particles, were then studied at fixed polymer concentration (2 wt\%) as a function of SDS concentrations, Figure 3. At low SDS concentration, the $R_{2 \text { sp }}$ of the latex and 2 wt\% HEUR mixtures show very subtle changes. The $R_{2 s p}$ has negative values at 3 wt\% SDS and higher concentrations, where macroscopic phase separation is observed as well.

The phase separation of the ternary mixture has been observed by Kostansek (27). The phase separation was correlated to depletion flocculation due to the interaction of HEUR with SDS. Hulden illustrated the absence of HEUR adsorption on to acrylic acid modified 
latex in presence of SDS at concentrations above the CMC due to the interaction of the SDS with the HEUR in solution (9). Therefore, the depletion flocculation is due to the presence of a non-adsorbing polymer/surfactant complex. However, depletion flocculation could occur due to the presence of free SDS micelles at high concentrations as demonstrated by Furusawa et al (31).

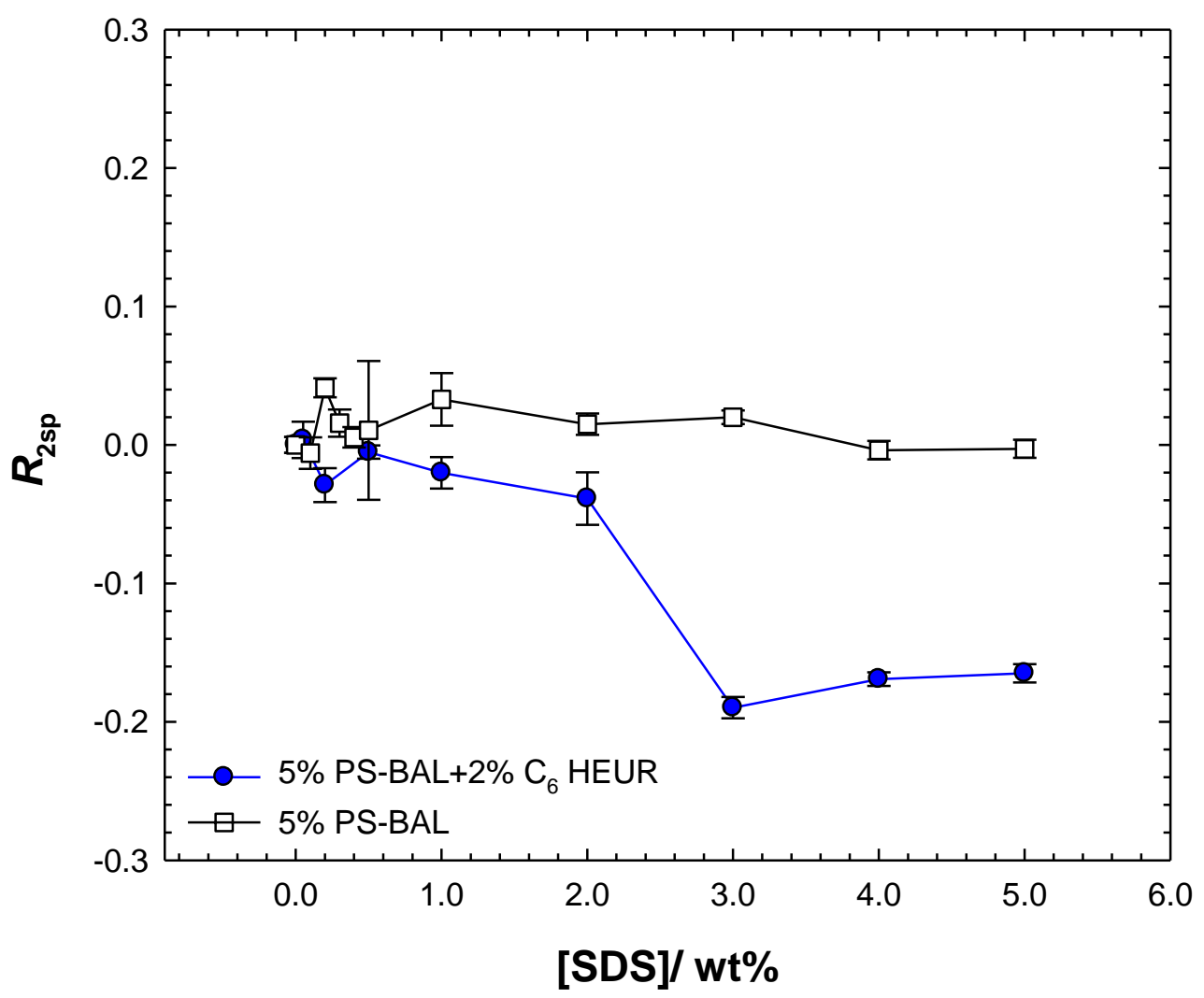

Figure 3. Specific relaxation rate $R_{2 s p}$ of the solvent in aqueous solutions containing $5 \mathrm{wt} \%$ PS-BA-L as a function of SDS in presence (circles) and absence (squares) of $2 \mathrm{wt} \% \mathrm{C}_{6}-\mathrm{L}-\left(\mathrm{EO}_{100}-\mathrm{L}\right)_{9}-\mathrm{C}_{6}$ in Hydroin buffered water, $\mathrm{pH}$ 9. The $R_{2 s p}$ of the mixtures are corrected relative to bare particle. Measurements were carried out at $25{ }^{\circ} \mathrm{C}$. The solid line is a guide for the eye. The error bars correspond to one standard deviation around the mean, derived from 5 repeat measurements on duplicate samples.

In this study, to differentiate the two potential mechanisms of depletion flocculation, $R_{2 s p}$ as a function of SDS in absence of polymer was recorded. The $R_{2 s p}$ of $5 \mathrm{wt} \%$ latex shows no significant changes as a function of SDS, Figure 3. Therefore, the observed depletion flocculation for the ternary system here is due to the presence of non-adsorbing HEUR/SDS complex.

Solution polymer aggregates in presence of PS-BA-L 
The choice of the polymer concentration (2wt\%) was dependent on the stability of the HEUR/PS-BA-L mixture determined in the solvent relaxation experiment. It is hypothesised that if there is a considerable level of bridging occurring, Figure 1-a, the diffusion of the polymer should be reflective of the state of aggregation, at least to a crude comparison of the simple polymer case. Therefore, measurements of polymer diffusion were conducted in presence and absence of the latex and the values of the polymer diffusion compared.

Figure 4, shows the self-diffusion coefficient of HEUR in presence and absence of PS-BAL. The polymer self-diffusion coefficients for the no-particle case have been taken from our previous study (32). One limiting case may be identified - that the polymer is completely adsorbed to the particle. In that case, the polymer diffusion would be comparable to the (mutual) diffusion coefficient of the PS-BA-L particles, that being calculated from the StokesEinstein equation based on the particle size measured here by DLS $\left(D=\frac{k_{B} T}{6 \pi r \eta}\right)$, where $D$ is the mutual diffusion coefficient of the particles, $k B$ Boltzmann constant, $T$ absolute temperature, $r$ radius of the particle and $\eta$ viscosity. The challenge in this case is what value to use for the viscosity, though the diffusion coefficient obtained by using the pure solvent viscosity is close to the observed value.

Rather, we compare the measured self-diffusion coefficient of the polymer solution in the absence and presence of the particles; as can be seen, figure 4, the HEUR diffusion $\left(D_{r}\right)$ is slightly slower in the presence of latex particles relative to HEUR on its own. Further, in the presence of SDS, the values of the $D_{r}$ approach unity, reminiscent of the simple polymer/surfactant behaviour. It is natural to conclude from these observations, that there is little polymer adsorbed to the particles. 


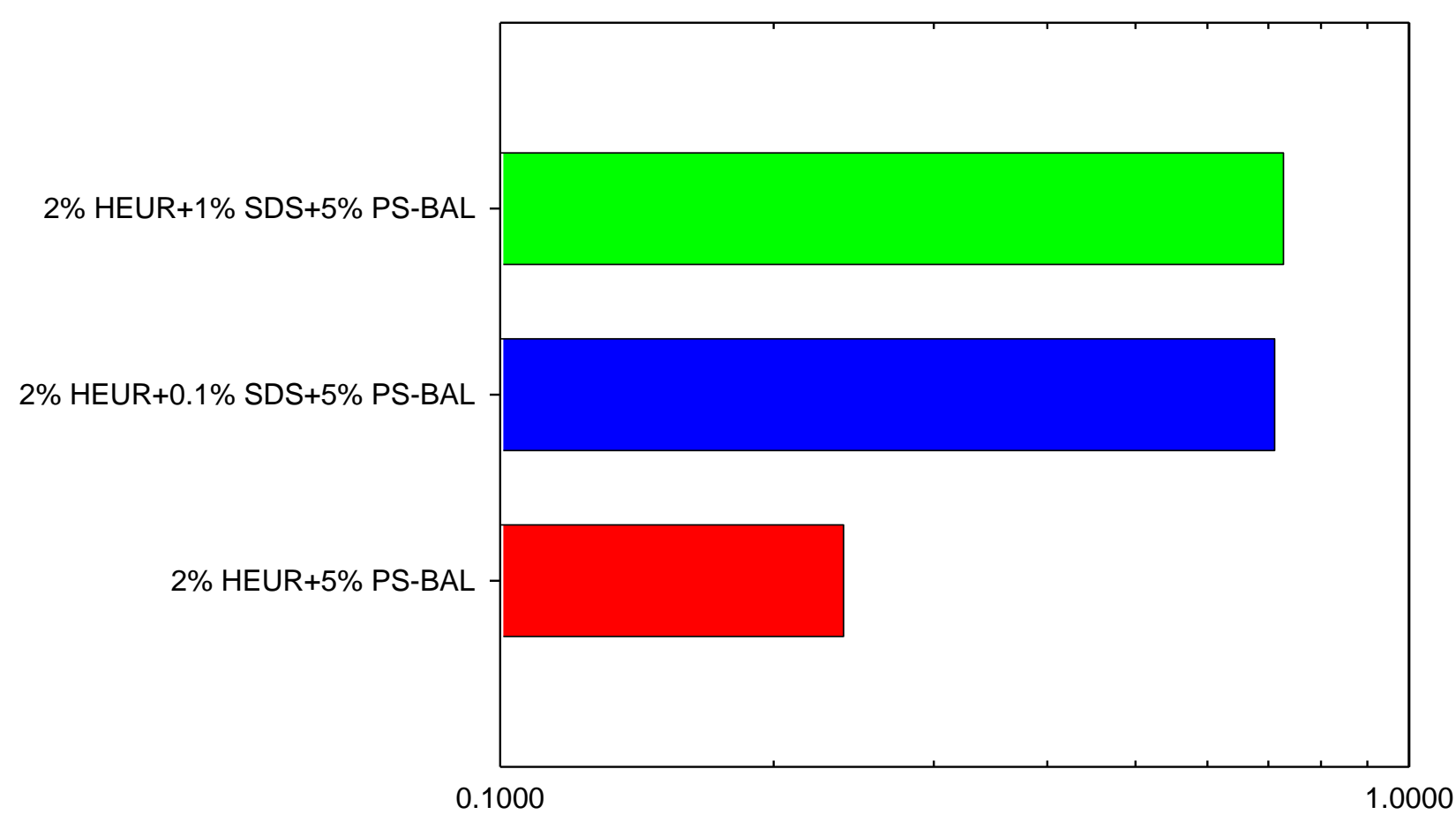

Relative diffusion of HEUR/SDS/PS-BAL mixtures

Figure 4. Diffusion coefficient of $\left.C_{6}-L-\left(E O_{100}-L\right)\right)_{9}-C_{6} / P S-B A-L$ and $C_{6}-L-$ $\left(\mathrm{EO}_{100}-\mathrm{L}\right)_{9}-\mathrm{C}_{6} / \mathrm{SDS} / \mathrm{PS}-\mathrm{BA}-\mathrm{L}$ mixtures relative to simple $\mathrm{C}_{6}-\mathrm{L}-\left(\mathrm{EO}_{100}-\mathrm{L}\right)_{9}-\mathrm{C}_{6}$ and $\mathrm{C}_{6}-\mathrm{L}-\left(\mathrm{EO}_{100}-\mathrm{L}\right) 9-\mathrm{C}_{6} / \mathrm{SDS}$, respectively. Samples were prepared in Hydroin buffered $\mathrm{D}_{2} \mathrm{O}, \mathrm{pH} 9$. Measurements were carried out at $25^{\circ} \mathrm{C}$.

In their NMR study of HEUR in the presence of PS-L, Uemura et al. (30) detected no signals from the PS-L, though the signal from the HEUR PEO backbone was evident. However, signals from the hydrophobes were not detected due to either their short $T_{2}$ or relatively low abundance. The adsorption of HEUR to PS-L was characterised by the observation of two diffusion coefficients, a faster one assigned to the non-adsorbed HEUR and a slower one to the adsorbed polymer. Crucially, the results of Uemura et al. are different to the results presented here where only one population is identified, figure S.3(a), this difference proposed to arise due to the weak adsorption of the HEUR to the latex particles in this study.

Beshah et al. [6] conducted a similar PGSE-NMR experiment in such a way to remove the signals of low molecular weight (fast diffusion) species, rather than having to deconvolute the contributions from the individual species in the sample. In solution, signals from the HEUR hydrophobes and the linkers were observed, but these disappeared on addition of hydrophobic latex. In the presence of hydrophillic latex, however, the signals from the linkers 
were observed, but those from the hydrophobes were still not evident, reflecting their adsorption to the particle surface. Here, likewise, neither the signal from the hydrophobes nor from the urethane linkers were observed neither in the absence nor presence of the particles indicating aggregation through these functional groups, Figure S.3(b\&c).

\section{SANS from HEUR in presence and absence of PS-BA-L}

To gain a better understanding of the HEUR conformation in presence of latex a partially deuterated PS-BA-L was synthesised to enable a contrast variation neutron scattering experiment. Figure 5 presents the observed scattering from a series of samples at a $\mathrm{H}_{2} \mathrm{O} / \mathrm{D}_{2} \mathrm{O}$ ratio that renders the particles "invisible". Evidence of this is the negligible scattering from the $3 \mathrm{wt} \%$ particle-only dispersion (triangles). Also shown, is the scattering from the simple $5 \mathrm{wt} \%$ polymer $\left(\mathrm{C}_{6}-\mathrm{L}-\left(\mathrm{EO}_{100}-\mathrm{L}\right) 9-\mathrm{C}_{6}\right)$ structure (circles). The remaining data set are mixtures of $5 \mathrm{wt} \%$ polymer and $0.5 \mathrm{wt} \%$ (squares), $3 \mathrm{wt} \%$ (triangles), or $5 \mathrm{wt} \%$ (hexagons) d-PS-h-BA-L.

Several points are striking in these data, and consistent with similar studies, e.g. (13). Firstly, at mid-Q where smaller length scales contribute to the scattering, the HEUR peak is invariant as a function of latex concentration. Secondly, at lower- $Q$, there is a significant decrease in the scattered intensity in the $0.5 \mathrm{wt} \% \mathrm{~d}$-PS-h-BA-L sample, which returns to a value close to the simple polymer with increasing latex concentration, i.e. at higher latex concentration (3 and 5 wt\% latex) the low-Q scattered intensity increases to be very close to the HEUR on its own. 


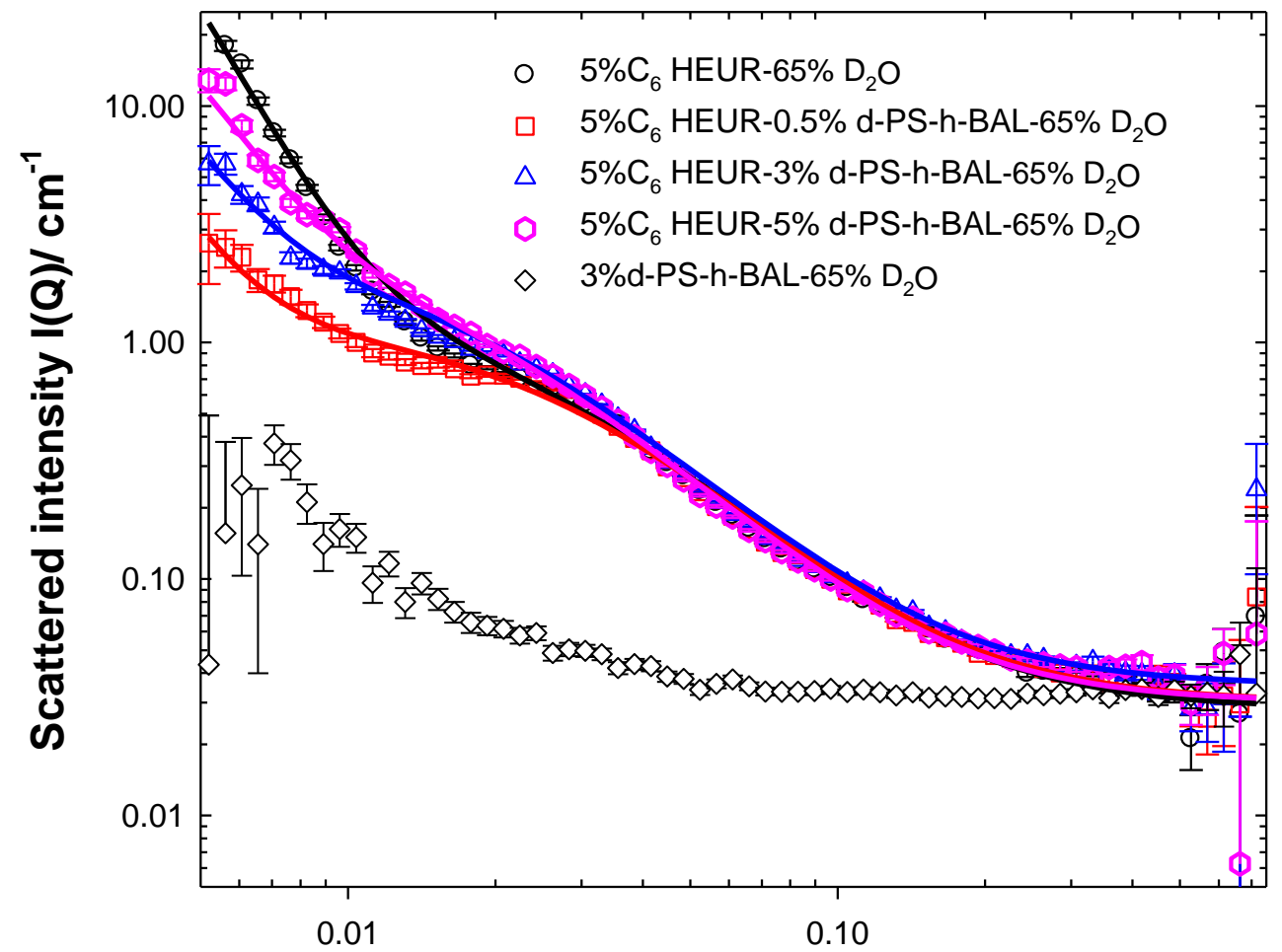

Scattering vector $Q / \AA^{-1}$

Figure 5. Small-angle neutron scattering from a series of polymer/particle blends; 5 wt $\%$ polymer in presence of 0.5 (squares), 3 (triangle), and $\mathbf{5}$ (hexagons) wt $\%$ d-PS-hBA-L, plus controls; 3 wt $\%$ d-PS-BA-L on match (diamonds), and 5 wt $\% \mathrm{C}_{6}$-L-(EO ${ }_{100}-$ L) $9-\mathrm{C}_{6}$ (circles) in $65 \% \mathrm{D}_{2} \mathrm{O} / \mathrm{H}_{2} \mathrm{O}$ mixture. Samples were prepared in Hydroin buffered solvents, $\mathrm{pH}$ 9. The solid lines are sphere and network model fits. The model is presented later in this section.

The peak present in HEUR scattering curve at $Q=0.03 \AA^{-1}$ represents the presence of an order in the sample coming from the polymer hydrophobic segments. The absence of changes in the intensity of HEUR peak as a function of latex particles at mid-Q indicates that only a few hydrophobic end-groups adsorb to the PS-BA-L surface, which do not disrupt the polymer hydrophobic aggregates arrangement in solution.

The change in scattered intensity at lower $Q$ reflects that changes in any larger length scale of the polymer may be due to the loss of the large-scale solution structure as a result of the formation of bigger aggregates which shifts the scattering intensity to lower $Q$. At higher concentration of particles, the increase of the intensity at low- $Q$ could be explained by particle aggregation and microscopic phase separation; however, this can be excluded as $R_{\text {2sp }}$ of the particles as a function of its concentration in presence and absence of $5 \mathrm{wt} \%$ HEUR is barely changing, Figure S.4. The polymer may rearrange in a manner similar to 
slightly higher polymer concentrations due to the volume restriction induced by latex addition at higher concentration.

Beaudoin et al. conducted a series of contrast variation experiments. In the first contrast the scattering contribution is from hydrophobically modified poly(ethylene oxide) (PEOM) (13). At low concentration, PEOM shows a peak at mid-Q which upon mixing with PS-L is replaced by a shoulder and shifted to lower $Q$, indicative of polymer adsorption to the latex particles. However, at higher polymer concentration the peak position and intensity of the polymer in the absence and presence of particles is unchanged; as the adsorbed fraction is negligible relative to total polymer concentration, in agreement with the results presented here. In the second contrast variation experiment, the scattering arose from particles only in polymer/particle mixtures (13). It was postulated that the particles are aggregating based on the d-spacing calculations.

In this paper, we have extended Beaudoin's et al. study to encompass a wider Q range to allow the detection of the changes in the intensity of the scattered intensity at lower $Q$, and in addition, taking the lead from Beaudoin et al. have developed a model with which to fit the scattering data.

Other researchers attempt to fit HEUR/latex mixtures to a spherical core-shell form factor with polydisperse core-radius and hard sphere structure factor for inter-particle repulsion (12). The fit captured the peaks at mid-Q, however, the changes at low- $Q$ were not captured. The inability of the model to capture the low- $Q$ features is due to the presence of the polymer network as shown in Figure 1. Here, several models to describe these data (sphere, coreshell, adsorbed layer) were tested but all were found to be deficient in some manner. Therefore, the data have been fitted to a compound model which captures all the features in the data.

$I(Q)=I(Q)_{1}\left\{\frac{4}{3} \pi R^{3} \frac{(\sin (Q R)-Q R \cos (Q R))}{(Q R)^{3}} S(Q)\right\}+\left\{\frac{I(Q)_{2}}{\left(1+Q^{2} \xi^{2}\right)}\right\}+\left\{\frac{I(Q)_{3}}{\left(1+Q^{2} A^{2}\right)^{2}}\right\}+B_{i n c}$

Equation 2

The first component of the compound fit with intensity $I(Q)_{1}$ is a sphere model to reflect the scattering from the structures formed by the polymer hydrophobes or the polymer hydrophobe/SDS aggregates. A charged structure factor is included here due to the SDS present in the HEUR/SDS mixed micelles, and is dependent on the inverse of the Debye 
screening length. The remaining terms with intensities $I(Q)_{2}$ and $I(Q)_{3}$ reflect the two correlation length network that the polymer may adopt ; a Lorentzian length $(\xi)$, the fluctuation in the polymer network structure, and a Debye-Bueche length $(A)$ postulated to be a length scale arising from the larger characteristic of the network. The Debye screening length was calculated from the sample concentration and ionic strength, and a backward calculation of volume fraction from the fit parameters used to test for fit validity. The values of the size of the polymer aggregates $(R)$, and two correlation lengths; $(\xi)$ and $(A)$ of the polymer were extracted from the fit, Table 2 .

From the fitting parameters, the sphere radius, intensity of the Lorentzian term, and the Lorentzian term are largely invariant with latex concentration. The Debye-Bueche intensity and term ' $A$ ' decreases as a function of latex concentration. Collectively, these observations may be explained by adsorption of the polymer to the latex particles and therefore a concomitant decrease in the spacing between the polymer hydrophobic aggregates.

\begin{tabular}{|c|c|c|c|c|}
\hline $\begin{array}{c}\text { Fit } \\
\text { parameters/ } \\
\text { Units }\end{array}$ & $5 \%$ HEUR & $\begin{array}{c}5 \% \text { HEUR } \\
+0.5 \% \text { latex }\end{array}$ & $\begin{array}{c}5 \% \text { HEUR } \\
+3 \% \text { latex }\end{array}$ & $\begin{array}{c}5 \% \text { HEUR } \\
+5 \% \text { latex }\end{array}$ \\
\hline $\begin{array}{c}\text { Intensity of } \\
\text { radius term }\end{array}$ & $7.2 \times 10^{-7}$ & $7.2 \times 10^{-7}$ & $7.2 \times 10^{-7}$ & $7.2 \times 10^{-7}$ \\
\hline Radius $(\AA)$ & $65 \pm 5$ & $65 \pm 5$ & $65 \pm 5$ & $65 \pm 5$ \\
\hline$C$ & 10 & 10 & 10 & 10 \\
\hline$l_{1}$ & 0.44 & 0.44 & 0.44 & 0.44 \\
\hline$\xi(\AA)$ & $25 \pm 1$ & $25 \pm 1$ & $25 \pm 1$ & $25 \pm 1$ \\
\hline$l_{2}$ & 325 & 325 & 325 & 5 \\
\hline$A(\AA)$ & $650 \pm 10$ & $600 \pm 10$ & $450 \pm 10$ & $80 \pm 10$ \\
\hline
\end{tabular}

\section{Table 2. SANS key parameters from the sphere and network model for the polymer and polymer/latex mixtures.}

The contrast match experiment HEUR/PS-BA-L, was replicated with the addition of two concentrations ( 0.1 and $1 \mathrm{wt} \%$ ) of $d / h$ SDS mixture to match the scattering length density (SLD) of the SDS to the solvent, hence the scattering contribution is from the polymer only. The scattering behaviour of the polymer in the ternary mixture is compared to the HEUR in HEUR/SDS complex and pure HEUR solutions.

The peak position $\left(Q=0.03 \AA^{-1}\right)$ of the polymer in the presence and absence of $0.1 \mathrm{wt} \% \mathrm{~d} / \mathrm{h}$ SDS does not show significant changes relative to the pure polymer solution, Figure S.5. 
The addition of PS-BA-L to solutions of polymer/SDS does not significantly change the intensity or position of the peak at mid-Q. However, a significant change in $\mathrm{C}_{6}-\mathrm{L}-\left(\mathrm{EO}_{100}-\mathrm{L}\right) \mathrm{g}$ $\mathrm{C}_{6} / \mathrm{SDS}$ scattering is observed at low-Q, similar to those changes observed in the polymer/latex mixtures in Figure 5.

In presence of $1 \mathrm{wt} \% \mathrm{~d} / \mathrm{h}$ SDS, the polymer peak position is shifted to higher $\mathrm{Q}$ range, Figure 6. The position of the polymer peak in the ternary mixture (HEUR/SDS/PS-BA-L) overlaps with the polymer/SDS complex rather than pure polymer peak. The same trend of scattering intensities at low-Q as a function of latex concentration in the presence of $0.1 \mathrm{wt} \%$ SDS is observed for the binary mixture (HEUR/PS-BA-L) and ternary mixture in the presence of 1 $\mathrm{wt} \%$ SDS. The overlapping of the polymer peak in presence of $1 \mathrm{wt} \%$ SDS and latex suggests that the SDS favourably interacts with the HEUR.

The main fitting parameters of the sphere and network model described earlier are presented for the 0.1 and $1 \mathrm{wt} \%$ SDS datasets in Tables S.1 and S.2, respectively. Similar trends observed for polymer/latex mixtures were noted here. From the fitting parameters, the sphere radius, Lorentzian intensity, and Lorentzian length do not change as a function of latex concentration. However, similar to the HEUR/latex mixture, the Debye-Bueche intensity, and length ' $A$ ' decrease as a function of latex concentration. The order of the decrease in the ' $A$ ' term and its intensity in the presence SDS, follow $1 \mathrm{wt} \%$ SDS $<0.1 \mathrm{wt} \%$ SDS $<0$ wt $\%$ SDS. These results suggest the weakening of the HEUR adsorption on addition of SDS. 


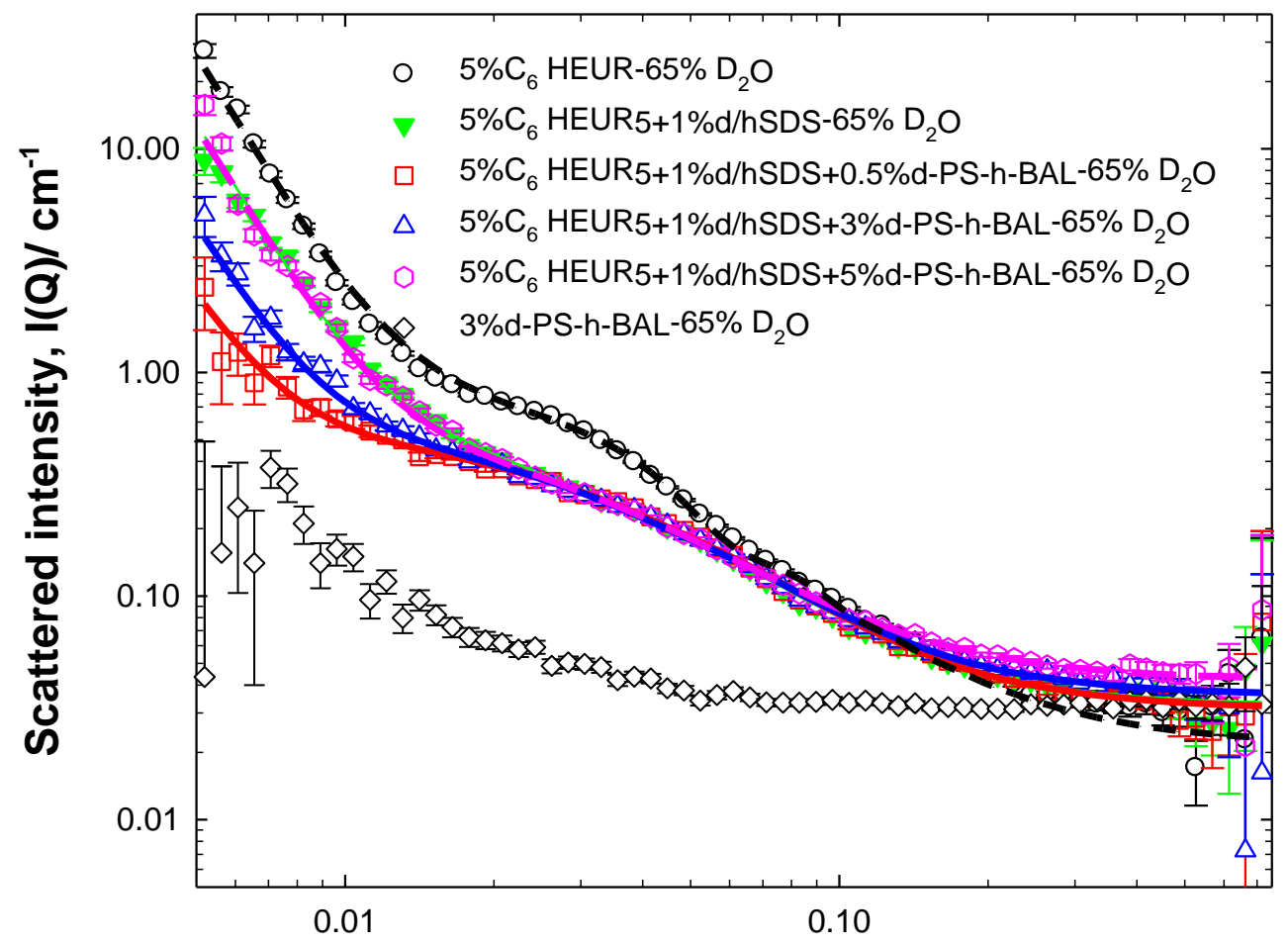

Scattering vector, $Q / \AA^{-1}$

Figure 6. Small-angle neutron scattering from a series of polymer/SDS/particle blends; $5 \mathrm{wt} \%$ polymer/1 wt\% SDS in presence of 0.5 (squares), 3 (triangles), and 5 (hexagons) wt $\%$ d-PS-h-BAL, plus controls; 3 wt $\%$ d-PS-BA-L on match (diamonds), 5 wt $\% \mathrm{C}_{6}-\mathrm{L}-\left(\mathrm{EO}_{100}-\mathrm{L}\right) 9-\mathrm{C}_{6}$ (circles), and 5 wt $\% \mathrm{C}_{6}-\mathrm{L}-\left(\mathrm{EO}_{100}-\mathrm{L}\right)_{9}-\mathrm{C}_{6} / 1 \mathrm{wt} \%$ SDS (triangles) in $65 \% \mathrm{D}_{2} \mathrm{O} / \mathrm{H}_{2} \mathrm{O}$ mixture. Samples were prepared in Hydroin buffered solvents, $\mathrm{pH}$ 9. The solid lines are sphere and network model fits.

Rheology of HEUR in absence and presence of PS-BA-L

In these experiments, the shear profile of the polymer at $5 \mathrm{wt} \%$ is measured as a function of latex concentration, where a shear independent viscosity is observed, Figure 7. Similar observations are noted for the $\mathrm{C}_{6}-\mathrm{L}-\left(\mathrm{EO}_{100}-\mathrm{L}\right){ }_{9}-\mathrm{C}_{6} / \mathrm{PS}-\mathrm{BA}-\mathrm{L}$ mixtures in presence 0.1 and 1 wt\% SDS, Figure 8. 


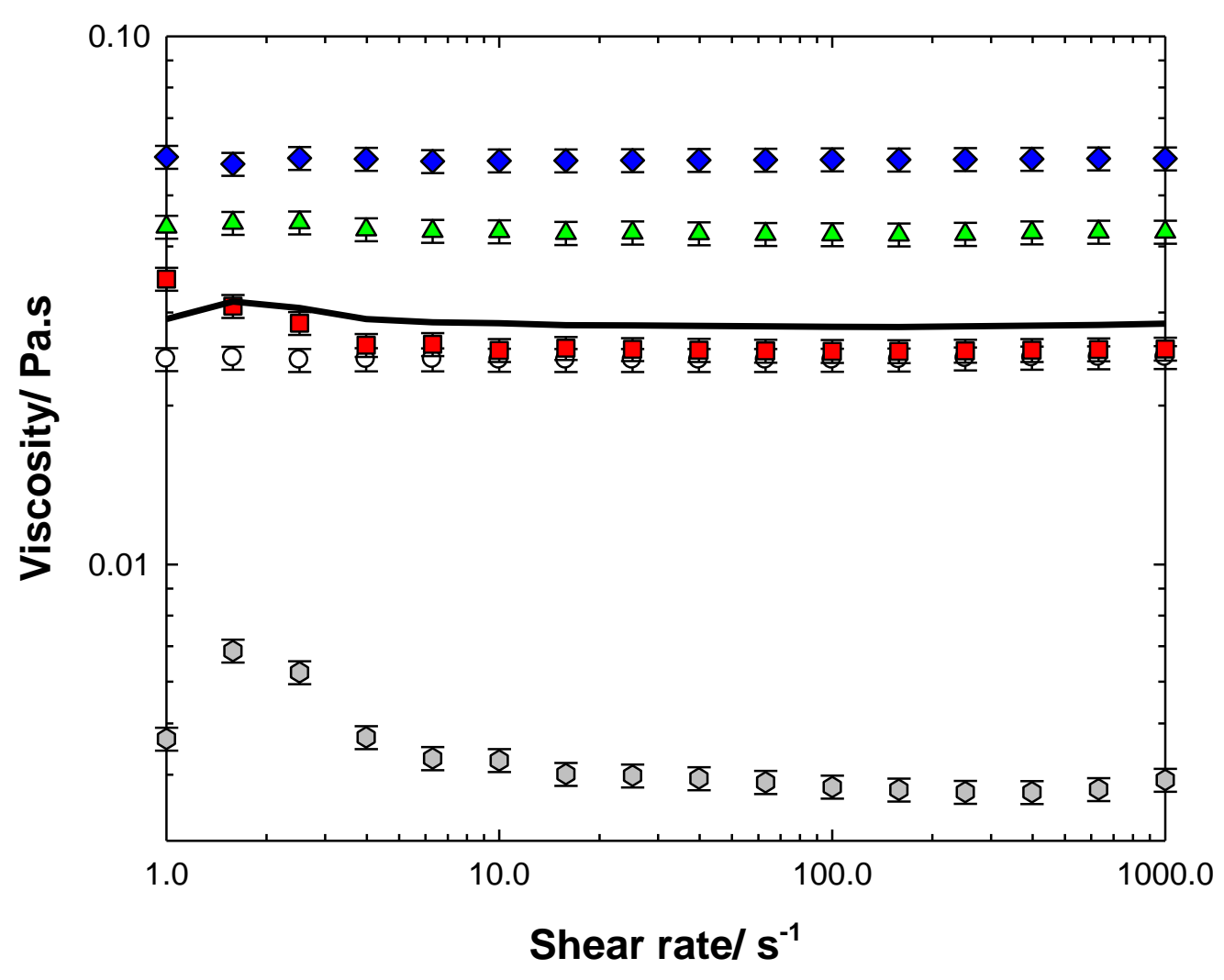

Figure 7. Shear profile of 5 wt $\%$ PS-BA-L (hexagon), 5 wt $\%$ HEUR in absence (circles) and presence of 0.5 (squares), 3 (triangles), 5 (diamonds) wt\% PSBA-L; linear addition of $5 \mathrm{wt} \%$ HEUR and $5 \mathrm{wt} \%$ PS-BA-L (black line). Samples were prepared in Hydroin buffer, $\mathrm{pH}$ 9. Measurements were carried out at $25{ }^{\circ} \mathrm{C}$. The error bars correspond to one standard deviation around the mean, derived from multiple repeat measurements recorded by the rheometer over the integration time of $5 \mathrm{~s}$. 


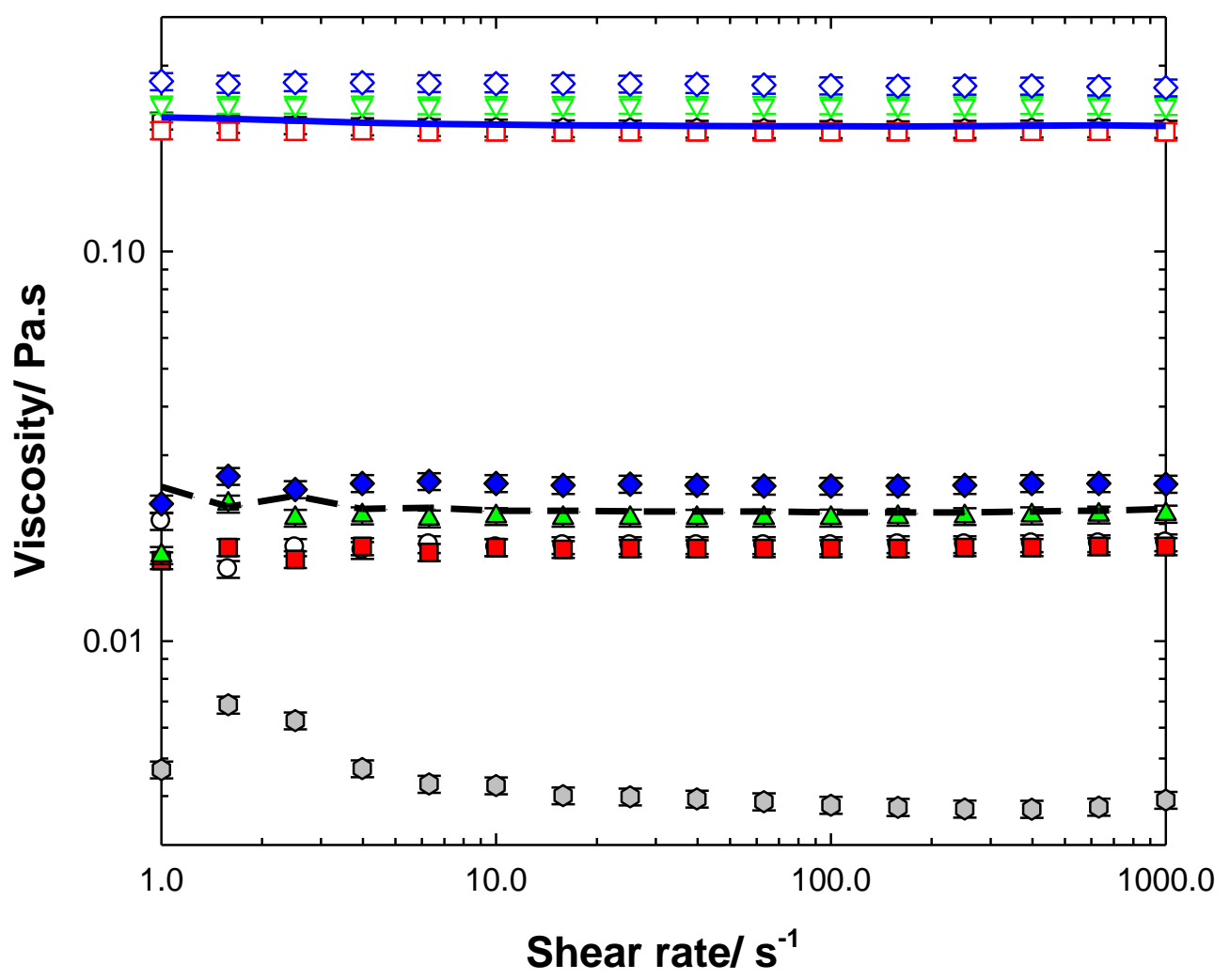

Figure 8. Shear profile of 5 wt $\%$ PS-BA-L (hexagon), 5 wt $\%$ HEUR in absence (circles) and presence of 0.5 (squares), 3 (triangles), and 5 (diamonds) wt $\%$ PS-BA-L at $0.1 \mathrm{wt} \%$ SDS (closed symbols) and $1 \mathrm{wt} \%$ SDS (open symbols). Linear addition $5 \mathrm{wt} \% \mathrm{C}_{6}-\mathrm{L}-\left(\mathrm{EO}_{100}-\mathrm{L}\right) 9-\mathrm{C}_{6} / \mathbf{0} .1 \mathrm{wt} \% \mathrm{SDS} / 5 \mathrm{wt} \%$ PS-BA-L (black dashed line), and 5 wt $\% \mathrm{C}_{6}-\mathrm{L}-\left(\mathrm{EO}_{100-\mathrm{L}}\right)_{9}-\mathrm{C}_{6} / 1 \mathrm{wt} \% \mathrm{SDS} / 5 \mathrm{wt} \%$ PS-BA-L (blue solid line). Samples were prepared in Hydroin buffered water, pH 9. Measurements were carried out at $25{ }^{\circ} \mathrm{C}$. The error bars correspond to one standard deviation around the mean, derived from multiple repeat measurements recorded by the rheometer over the integration time of $5 \mathrm{~s}$.

The relative viscosities $\left(\eta_{r}\right)$ over a range of shear rates $\left(10-500 \mathrm{~s}^{-1}\right)$ of the $\mathrm{C}_{6}-\mathrm{L}-\left(\mathrm{EO}_{100}-\mathrm{L}\right){ }^{9-}$ $\mathrm{C}_{6} / \mathrm{PS}-\mathrm{BA}-\mathrm{L}$ mixtures (relative to the polymer $\left(\eta_{r}=\frac{\eta_{\text {mixture }}}{\eta_{\text {polymer }}}\right)$ ) and $\mathrm{C}_{6}-\mathrm{L}-\left(\mathrm{EO}_{100} \mathrm{~L}\right)$ g- $^{-}$ $\mathrm{C}_{6} / \mathrm{SDS} / \mathrm{PS}-\mathrm{BA}-\mathrm{L}$ (relative to the polymer/SDS mixture, $\eta_{r}=\frac{\eta_{\text {ternarymixture }}}{\eta_{\text {polymer } / S D S}}$ ), were then plotted as a function of latex concentration, Figure 9. The viscosity of both mixtures increase with latex concentration. The increase in both relative viscosities are not additive (i.e. $\left.\eta_{\text {mixture }} \neq \eta_{\text {polymer }}+\eta_{\text {dispersion }}\right)$, reflective of synergistic increases due $\mathrm{C}_{6}-\mathrm{L}-\left(\mathrm{EO}_{100-}\right.$ $\mathrm{L}) 9-\mathrm{C}_{6}$ adsorption. However, in the presence of increasing amounts of SDS, the increase in viscosity associated with the addition of the latex is greatly reduced - the relative viscosity as a function of latex concentration displays a shallower slope with increasing SDS - 
reflecting the weakening of the interaction between the polymer and latex in presence of SDS.

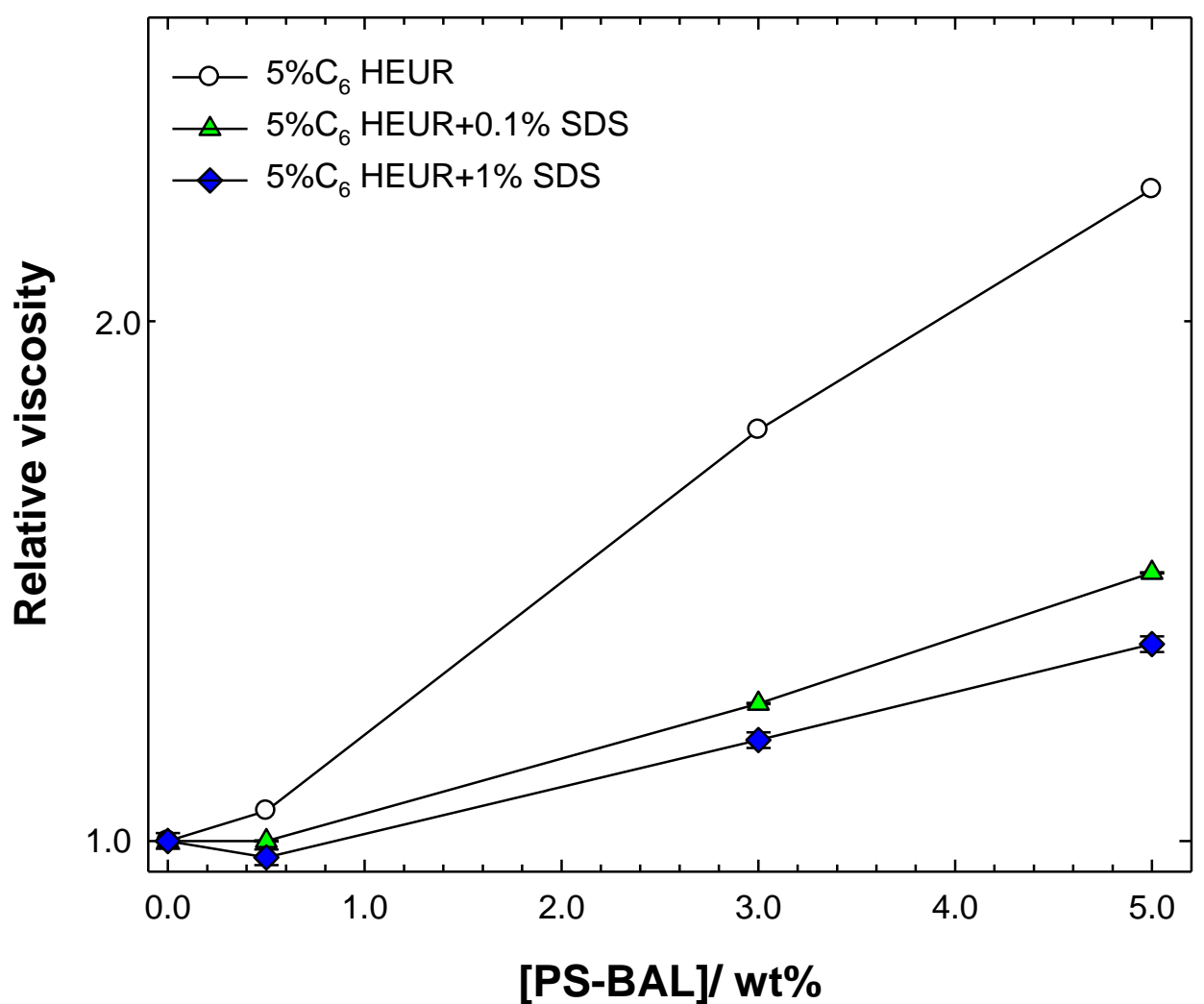

Figure 9. Average relative viscosities over a range of shear rates (10 - 500 $\mathrm{s}^{-1}$ ) of $\mathrm{C}_{6}-\mathrm{L}-\left(\mathrm{EO}_{100}-\mathrm{L}\right){ }^{9-} \mathrm{C}_{6} / \mathrm{PS}-\mathrm{BA}-\mathrm{L}$ mixtures in absence (circles), and presence of 0.1 (triangles), and 1 (diamonds) wt\% SDS. Samples were prepared in Hydroin buffered water, $\mathrm{pH} 9$. Measurements were carried out at $25{ }^{\circ} \mathrm{C}$. The solid lines are guides for the eye. The error bars correspond to mean values derived from the standard deviations associated with the raw data presented in the earlier figures.

\section{Effect of latex hydrophobicity}

Some selected experiments from the HEUR/PS-BA-L system have been repeated with a less hydrophobic latex. For brevity, the results only are presented here but the analyses are included in the supplemental section. The $R_{2 s p}$ curve for the HEUR/AA-PS-BA-L overlaps the simple HEUR curve, indicating the absence of adsorption, Figure S.6. The absence of phase separation in the HEUR/AA-PS-BA-L allowed measurement of the size of the particles under conditions where excess polymer would preclude such a calculation; no appreciable change in particle size upon mixing $\mathrm{C}_{6}-\mathrm{L}-\left(\mathrm{EO}_{100}-\mathrm{L}\right){ }_{9}-\mathrm{C}_{6}$ with the acrylic acid latex AA-PS-BA-L, Figure S.7 was observed. The self-diffusion cofficient of the polymer measured by NMR also showed no change upon the addition of the particle, Figure S.8. All these 
results strongly suggest the absence of adsorption of the polymer to the less hydrophobic particle.

\section{Conclusion}

The interaction of HEUR and latex is complex and dependent on many factors (e.g. latex surface chemistry, size, polymer architecture, and concentration). In this paper, the interaction of a HEUR with latices of different surface chemistries have been studied in the presence and absence of SDS. The use of the more hydrophilic latex AA-PS-BA-L shows no evidence of HEUR adsorption to the latex surface; evidenced by the absence of enhancement of the $T_{2}$ (spin-spin relaxation times) of the solvent in of HEUR/AA-PS-BA-L mixtures, no change in the particle size of the latex on addition of polymer, and no change in the self-diffusion coefficient of the HEUR. On the contrary, there is a weak interaction between the HEUR and the more hydrophobic latex PS-BA-L evidenced by the changes in the relaxation time of the solvent (a measure of the surface area of the particles) attributed to bridging flocculation at low polymer concentrations. An increase in the HEUR solution viscosity as a function of latex concentration and a decrease in the polymer self-diffusion coefficient upon addition of latex also supports the presence of an interaction. In addition, changes in the polymer larger scale length (bridging polymer chains) have been observed in the SANS data. The presence of only weak adsorption of $\mathrm{C}_{6}-\mathrm{L}-\left(\mathrm{EO}_{100}-\mathrm{L}\right){ }_{9}-\mathrm{C}_{6} \mathrm{HEUR}$ to the particles may be due to a preferential (self-)aggregation in solution or the adoption of a highly extended conformation at the particle surface on account of the short size of the hydrophobic end-groups of the polymer. In the $\mathrm{C}_{6}-\mathrm{L}-\left(\mathrm{EO}_{100}-\mathrm{L}\right){ }_{9}-\mathrm{C}_{6} / \mathrm{AA}-\mathrm{PS}-\mathrm{BA}-\mathrm{L}$ mixtures, the model presented by Pham et al. (10) seems to better describe this system compared to the newer model proposed by Beshah et al. (6); though this most likely reflects a greater association of the polymer hydrophobes in solution.

The addition of SDS to polymer/latex mixtures further weakened the adsorption of the polymer to the latex, evidenced by the similarity of several characteristics of the system (polymer self-diffusion coefficient, viscosity, relaxation time of the solvent) as a function of latex concentration. 


\section{Acknowledgment}

We would like to thank the UK science and technology facilities council (STFC) for beam time allocation in ISIS (RB1710213, 1620114, 1620112). Thanks are also due to the University of Greenwich and AkzoNobel for financial support. 


\section{References}

1. Holmberg K. Handbook of Surface and Colloid Chemistry. Fourth Edition. Florida, USA:CPR Press; 2015.

2. Aulton ME. Pharmaceutics-The Science of Dosage from Design. Second Edition. New York, USA:Churchill Livingstone; 2002.

3. Panrat K, Boonme P, Taweepreda W, Pichayakorn W. Formulations of Natural Rubber Latex as Film Former for Pharmaceutical Coating. Procedia Chem. 2012;4:322-327.

4. Richey B, Kirk AB, Eisenhart EK, Fitzwater S, Hook J. Interactions of associative thickeners with paint components as studied by the use of a fluorescently labeled model thickener. J Coatings Thechnol. 1991;63(798):31-40.

5. Glass JE. Adsorption of hydrophobically-modified, ethoxylated urethane thickeners on latex and titanium dioxide disperse phases. Adv Colloid Interface Sci. 1999;79(2):123-148.

6. Beshah K, Izmitli A, Van Dyk AK, Rabasco JJ, Bohling J, Fitzwater SJ. Diffusionweighted PFGNMR study of molecular level interactions of loops and direct bridges of HEURs on latex particles. Macromolecules. 2013;46(6):2216-2227.

7. Quadrat O, Horsky J, Snuparek J. Thickening effect of commercial associative thickeners on the latices of copolymers of acrylic monomers carrying hydrophilic reactive groups. J Dispersion Sci Technol. 2003;24(2)179-184.

8. Ou-yang HD, Gao Z. A pancake-to-brush transition in polymer adsorption. J Phys II. 1991;1(11):1375-1385.

9. Hulden M. Hydrophobically modified urethane-ethoxylate (HEUR) associative thickeners 2. Interaction with latex. Colloids Surfaces A Physicochem Eng Asp. 1994;88(2-3):207-221.

10. Pham QT, Russel WB, Lau W. The effects of adsorbed layers and solution polymer on the viscosity of dispersions containing associative polymers. $J$ Rheol. 1998;42(1)159-176.

11. Santos FA, Bell TJ, Stevenson AR, Christensen DJ, Pfau MR, Nghiem BQ, Kasprzak $\mathrm{CR}$, Smith TB, Fernando RH. Syneresis and rheology mechanisms of a latex-HEUR 
associative thickener system. J Coatings Technol Res. 2017;14(1):57-67.

12. Chatterjee T, Nakatani AI, Dyk AKV. Shear-dependent interactions in hydrophobically modified ethylene oxide urethane (HEUR) based rheology modifier-latex suspensions: Part 1. Molecular microstructure. Macromolecules. 2014;47(3):11551174.

13. Beaudoin E, Lapp A, Hiorns RC, Grassl B, François J. Neutron scattering of hydrophobically modified poly(ethylene oxide) in aqueous solutions in the presence of latex particles. Polymer. 2002;43(9):2677-2689.

14. Pisarcik M, Bakos D, Ceppan M. Interactions of latex spheres and anionic surfactant in hydrophobically modified polymer aqueous solution. Colloids Surf, A Physicochem Eng Asp. 1993;81:161-166.

15. Lauten RA, Kjoniksen A, Nystrom B. Adsorption and desorption of unmodified and hydrophobically modified ethyl(hydroxyethyl)cellulose on polystyrene latex particles in the presence of ionic surfactants using dynamic light scattering. Langmuir. 2000;16(10):4478-4484.

16. Lauten RA, Kjoniksen A, Nystro B. Colloid polymer interactions and aggregation in aqueous mixtures of polystyrene latex, sodium dodecyl sulfate, and a hydrophobically modified polymer : A dynamic light scattering study. Langmuir. 2001;17(3):924-930. $2001 ;(3): 924-30$.

17. Mahli DM, Steffenhagen MJ, Xing L, Glass JE. Surfactant behavior and its influence on the viscosity of associative thickeners solutions, thickened latex dispersions, and waterborne latex coatings. J Coat Technol. 2003;75(938):39-51.

18. Winnik MA, Yekta A. Associative polymers in aqueous solution. Curr Opin Colloid Interface Sci. 1997;2(4):4234-4236.

19. Alami E, Almgren M, Brown W. Interaction of hydrophobically end-capped poly(ethylene oxide) with nonionic surfactants in aqueous solution. Fluorescence and light scattering studies. Macromolecules. 1996;29(14):5026-5035.

20. Zhang K, Xu B, Winnik MA, Macdonald PM. Surfactant Interactions with HEUR Associating Polymers. J Phys Chem. 1996;100(23):9834-9841.

21. Hulden M. Hydrophobically modified urethane-ethoxylate (HEUR) associative thickeners 1. Rheology of aqueous solutions and interactions with surfactants. 
Colloids Surf, A Physicochem Eng Asp. 1994;82(3):263-277.

22. Ma Z, Chen M, Glass JE. Adsorption of nonionic surfactants and model HEUR associative thickeners on oligomeric acid-stabilized poly(methyl methacrylate) lattices. Colloids Surf, A Physicochem Eng Asp. 1996;112(2):163-184.

23. Abrahmsen-Alami S, Stilbs P. NMR Self-Diffusion of Associative Polymers in Aqueous Solution: The Influence of the Hydrocarbon End-Chain Length on the Polymer Transport Dynamics in Single-and Two-Component Mixtures. J Colloid Interface Sci. 1997;189(189):137-43.

24. Claridge. High-Resolution NMR Techniques in Organic Chemistry. Second. Oxford, UK: ELSVIERE; 2009.

25. Heenan RK, King SM, Turner DS, Treadgold JR. SANS2d at the ISIS Second Target Station. 17th Meet Int Collab Adv Neutron Sources. 2005;1-6. Available from: http://www.isis.stfc.ac.uk/instruments/sans2d/publications/sans2d-at-isis10323.pdf, (accessed November 2016).

26. Cooper CL, Cosgrove T, Duijneveldt JS, Murray M, Prescott SW. The use of solvent relaxation NMR to study colloidal suspensions. Soft Matter. 2013;9(30):7211-7228.

27. Kostansek E. Using Dispersion/flocculation phase diagrams to visualize interactions of associative polymers, latexes, and surfactants. J Coat Technol. 2003;75(940):1-8.

28. Reuvers AJ. Control of rheology of water-borne paints using associative thickeners. Prog Org Coatings. 1999;35:171-181.

29. Jenkins RD, Durali M, Silebi CA, El-Aasser MS. Adsorption of model associative polymers on monodisperse polystyrene latex. J Colloid Interface Sci. 1992; 154(2):502-521.

30. Uemura Y, Macdonald PM. Associating polymer binding to polystyrene latex. Macromolecules. 1996;29(1):63-69. .

31. Furusawa K, Sato A, Shirai J, Nashima T. Depletion flocculation of latex dispersion in ionic micellar systems. J Colloid Interface Sci. 2002;253:273-2788.

32. Ibrahim MS, Valencony J, King S, Murray M, Szczygiel A, Alexander BD, Griffiths PC. Studying the interaction of hydrophobically modified ethoxylated urethane (HEUR) polymers with sodium dodecylsulfate (SDS) in concentrated polymer solutions. $J$ 
Colloid Interface Sci . 2018;529:588-598. 